\title{
Food induced stimulation of the antisecretory factor can improve symptoms in human inflammatory bowel disease: a study of a concept
}

\author{
S Björck, I Bosaeus, E Ek, E Jennische, I Lönnroth, E Johansson, S Lange
}

Department of Surgery, Lundby Hospital, Sweden S Björck

Department of Clinical Nutrition, Göteborg University, Göteborg, Sweden

I Bosaeus

Department of Clinical Bacteriology, Göteborg University, Göteborg,

Sweden

E Ek

S Lange

Department of Anatomy and Cell Biology, Göteborg University, Göteborg, Sweden

E Jennische

Department of Medical Microbiology and Immunology, Göteborg University, Göteborg, Sweden I Lönnroth

E Johansson

Correspondence to: Dr S Lange, Department of Clinical Bacteriology, Guldhedsgatan 10, S-413 46 Göteborg, Sweden. Email: stefan.lange@microbio.gu.se

Accepted for publication 25 November 1999

\begin{abstract}
Background-Antisecretory factor (AF), a $41 \mathrm{kDa}$ cloned and sequenced protein, suppresses intestinal inflammation and hypersecretion in animals. Endogenous AF production can be induced by dietary modifications in several animal species, and this feed has been shown to reduce the incidence of diarrhoeal disease in weaning piglets. The role of $\mathrm{AF}$ in intestinal disease in humans is not known.

Aims-To study the effects of hydrothermally processed cereals, optimised for AF induction in animals, added to the diet of patients with longstanding symptoms of inflammatory bowel disease (IBD).

Patients-Fifty three patients with IBD (ulcerative colitis and Crohn's disease) were entered into the study, and 50 completed follow up. The experimental group consisted of 16 females (mean age 50 (SEM 5) years) and 10 males (41 (4) years) and the placebo group of 12 women (41 (4) years old) and 12 men (51 (5) years).

Methods-Patients were randomised to receive either hydrothermally processed cereals (active treatment) or the same amount of ordinary cereals (placebo treatment) for four weeks in a double blind study design. Baseline diet and medications remained unchanged. Bowel symptoms, plasma levels of AF, and colonic biopsies were evaluated before and after treatment.
\end{abstract}

Results-The active treatment significantly improved subjective ratings of clinical symptoms and increased plasma AF levels compared with placebo. Plasma lipid levels were unaffected.

Conclusion-Hydrothermally processed cereals can induce AF production in human IBD. This increase in endogenous AF activity is associated with clinical improvement. Further studies are warranted to clarify the exact role of $A F$ in human intestinal disease.

(Gut 2000;46:824-829)

Keywords: antisecretory factor; functional food; ulcerative colitis; Crohn's disease

We have isolated, cloned, and sequenced "antisecretory factor" (AF), a $41 \mathrm{kDa}$ protein. ${ }^{1}$ $\mathrm{AF}$ exerts an inhibitory effect on various experimental diarrhoea models in the rat and pig small intestinal ligated loops. Thus only
$10^{-11} \mathrm{M}$ to $10^{-12} \mathrm{M}$ of recombinant $\mathrm{AF}$ is required to revert intestinal hypersecretion in vivo when added from both the mucosal and the serosal side of the epithelium. Recombinant $\mathrm{AF}$ also inhibits the inflammatory response induced by toxin A from Clostridium difficile in rat jejunal loops. ${ }^{2}$

Over the past decade we have been working in close cooperation with European farmers on improvement in feed quality for meat producing animals. It has been proved that optimally designed feed reduces diarrhoea and increases daily weight gain in newly weaned pigs better than that achieved by traditional high protein feed supplemented with antibiotics. ${ }^{3}$ AF has proved to be one of the most useful laboratory variables in the design of optimal feed composition for livestock. ${ }^{4}$

In the present study we evaluated food optimised for AF induction in humans suffering from intestinal disease. Thus patients with a clinical condition associated with pathological intestinal secretion and/or intestinal inflammation were selected. A double blind study was designed in patients with inflammatory bowel disease (IBD) (ulcerative colitis or Crohn's disease). Two criteria had to be fulfilled: (1) patients should have a longstanding history (more than two years) of intestinal dysfunction and a diagnosis of ulcerative colitis or Crohn's disease, but should be in a stable clinical condition making outpatient treatment possible and; (2) conventional medical treatment and the patient's normal food habits should not be changed.

Patients were randomised to one of two groups: group A, experimental group, received hydrothermally processed cereals capable of increasing plasma AF levels (active food); group B, control group, received the same type of food but the hydrothermally processed cereals were replaced by ordinary cereals without specific $\mathrm{AF}$ inducing properties (placebo food). Active and placebo food had the same taste, appearance, and content of energy and nutrients.

The biological role of AF in human intestinal diseases is largely unknown. Thus we hypothesised that the active food would induce AF and affect the clinical outcome of intestinal disease in humans. Consequently, the investigation was designed to study a concept (that is, to evaluate if an increase in endogenous $\mathrm{AF}$ in

Abbreviations used in this paper: $\mathrm{AF}$, antisecretory factor; VAS, visual analogue scale; IBD, inflammatory bowel disease; CRP, $\mathrm{C}$ reactive protein. 
patients suffering from IBD could affect the clinical outcome of the disease).

\section{Material and methods}

PATIENTS AND METHODS

The study design was approved by the human ethics committee of Göteborg University. Patients subjected to the active treatment (experimental group) received a diet supplemented with hydrothermally processed cereals capable of endogenous AF stimulation. The control group was given the same type of food but the hydrothermally processed cereals were replaced by ordinary cereals (placebo group). A control panel, responsible for food production, ensured that no difference in taste or quality could be detected between the active and control foods. A delivery firm ensured that the correctly coded cereal product was delivered at the home of the participants. The code of the study was kept at the Swedish "Notarius Publicus" and was not broken until all of the clinical and laboratory results had been registered.

Patients attending an outpatient gastroenterological clinic (head of clinic, SB) with a diagnosis of ulcerative colitis or Crohn's ileitis or colitis were invited to participate in the study. All participating patients had longstanding ( $>2$ years) gastrointestinal disorders resulting in loose stools or frank diarrhoea, and an abnormal number of bowel movements each day, despite conventional medical treatment. All patients had previously undergone biopsies of the rectal mucosa, and the histology of these biopsies verified the clinical diagnosis of ulcerative colitis or Crohn's disease. Thus the selection of patients fulfilled the criteria of Truelove and Witts.

Currently, approximately 500 patients with IBD attend the clinic. All 53 eligible patients agreed to take part in the study: 50 patients had chronic ulcerative colitis (29 total colitis, 21 distal colitis) and three patients had Crohn's disease (one ileal disease, two colonic disease). Patients were randomly assigned a daily intake of products containing a certain amount of either hydrothermally treated cereals (active food, capable of endogenous AF stimulation, experimental group) or ordinary cereals (placebo food, without measurable effects on endogenous AF synthesis). Patients were instructed to have a daily intake of $1 \mathrm{dl}$ of muesli, $100 \mathrm{~g}$ of pasta cooked for 4-5 minutes, and a roll. They were also informed that one roll was equal to $1 \mathrm{dl}$ of muesli or $50 \mathrm{~g}$ of pasta.

All patients reported that they replaced a major part of their ordinary cereal intake with the cereal food used in the study. However, neither the overall diet nor the time schedule for daily food consumption was affected by cereal intake during the test period.

The cereals of the active food were treated hydrothermally in a process similar to malting. The hydrothermal process was continued until the content of sugars and amino acids of the products were in the range given in table 1 . After processing, the cereals were dried to $10 \%$ moisture content. Sugar content was assayed by soaking $5 \mathrm{~g}$ of cereal products for 10
Table 1 Content of sugars and amino acids in the cereals of the active food before and after the hydrothermal process. The hydrothermal process was continued until the content of sugars and amino acids of the products were in the ranges given

\begin{tabular}{lll}
\hline Content & $\begin{array}{l}\text { Before the process } \\
(\mathrm{mg} / \mathrm{g} D M)\end{array}$ & $\begin{array}{l}\text { After the process } \\
(\mathrm{mg} / \mathrm{g} D M)\end{array}$ \\
\hline Glucose & $0.3-0.4$ & $0.6-5.3$ \\
Fructose & $0.3-0.4$ & $0.6-3.1$ \\
Sucrose & $8.4-14.4$ & $36.8-65.7$ \\
Maltose & 0.0 & $4.0-9.0$ \\
Histidine & 0.0 & $0.06-0.25$ \\
Glutamic acid & $0.12-0.20$ & $0.42-0.44$ \\
Lysine & $0.03-0.06$ & $0.15-0.29$ \\
Tryptophan & $0.09-0.22$ & $0.28-0.45$ \\
Isoleucine & 0.0 & $0.05-0.30$ \\
\hline
\end{tabular}

minutes at room temperature in $100 \mathrm{ml}$ of a buffer consisting of $20 \mathrm{mM} \mathrm{Na} \mathrm{HPO}_{4}, 6 \mathrm{mM}$ $\mathrm{NaCl}, \mathrm{pH}$ 7. Samples were taken from the solution and analysed for sugars and amino acids by HPLC and standard procedures.

Both active and placebo food had the same energy and nutrient content. The food consisted of muesli, rolls, and frozen fresh pasta and was produced by BioDoc AB (Box 30 192, S-104 25 Stockholm, Sweden).

PATIENT REGISTRATION

All patients kept a diary over a six week period noting the number of bowel movements per day and the consistency of the stools (hard, solid, loose, or watery). At the end of the study each patient and the principal investigator (SB) evaluated the effect of the added cereal diet on the patient's bowel habits. Clinical improvement or clinical deterioration was plotted as a percentage on a visual analogue scale (VAS score) and expressed as percentage change. In principle, this implies that a score of $100 \%$ or more represents total normalisation of intestinal function whereas a score on the minus part of the scale is correlated with subjective deterioration of a multitude of clinical functions related to the intestine. There was good correlation between patient registrations in the diaries and VAS scores.

\section{CLINICAL EXAMINATION}

All patients were thoroughly investigated by endoscopy, biopsies, radiography, blood tests, stool cultures, and malabsorption tests at least six months before the start of the study. Most patients had suffered from their disease for several years (minimum two years, maximum 25 years). Endoscopy with biopsies was performed in each patient at the beginning of the study and at the end of the four week period of the active or placebo diet. All biopsies were obtained in a standardised way, $10 \mathrm{~cm}$ proximal to the linea dentata in the posterior wall of the rectum.

Plasma was collected from each patient just before start of the study and at weeks 4 and 8 of the study period. Plasma samples were stored at $-20^{\circ} \mathrm{C}$ until tested. Some of the patients underwent left sided colonoscopy at the end of the study.

\section{HISTOLOGY}

All rectal biopsies were taken after obtaining informed consent from patients, and were 
carefully selected via the endoscope. The biopsies were immersed in $4 \%$ paraformaldehyde in phosphate buffered saline (PBS $145 \mathrm{mM}$, $\mathrm{NaCl}$ buffered to $\mathrm{pH} 7.2$ with $70 \mathrm{mM}$ $\mathrm{Na}_{2} \mathrm{PO}_{4}$ ), frozen in liquid nitrogen, and $7 \mu \mathrm{m}$ thick cryostat sections were prepared. The sections were first incubated with the affinity purified antiserum against the $\mathrm{C}$ terminal of the $\mathrm{AF}$ protein ${ }^{1}$ diluted $1 / 600$ and then with alkaline phosphatase conjugated swine antirabbit immunoglobulins (Boehringer Mannheim). The immune reaction was visualised by incubating the sections in a substrate solution containing nitroblue tetrazolium and 5-bromo-4-chloro3-indolyl phosphate tolodium salt (Boehringer Mannheim). Serial sections were stained with periodic acid-Schiff stain (PAS) and haematoxylin.

To classify AF positive cells, staining of serial sections from the biopsies with various $\mathrm{T}$ cell antisera (CD3, CD4, CD8, CD20, CD45RO, and CD68, results not shown) was performed. Thus the position in the tissue in addition to the gross morphology of the AF positive cells were compared with the same markers for the cells appearing positive with the various $T$ cell antisera. The results indicated that the $\mathrm{AF}$ positive cells were either CD4 or CD8 positive, and that AF cells could tentatively be classified as a subgroup of $\mathrm{T}$ cells.

PREPARATION OF AF AND TEST OF AF POTENCY $\mathrm{AF}$ was purified from plasma using affinity chromatography as previously described. ${ }^{1}$ In brief, after passage of the plasma through a small agarose column (Sepharose 6B, Pharmacia LKB Biotechnology, Stockholm, Sweden), the agarose adsorbed AF was eluted with $1 \mathrm{M}$ methyl- $\alpha-\mathrm{D}$-glucoside. The eluate was dialysed against PBS for 24 hours at $4^{\circ} \mathrm{C}$ and then stored at $-20^{\circ} \mathrm{C}$ until use.

The potency of the purified AF was determined by the ligated loop assay in rats using cholera toxin as a secretagogue. ${ }^{6}$ In brief, under ether anaesthesia, one loop, about $10 \mathrm{~cm}$ in length, was made in the jejunum. The AF test substance $(2 \mathrm{ml})$, prepared from the plasma of the patients as described above, was administrated intravenously via the dorsal vein of the penis. The loop was then challenged with $3 \mu \mathrm{g}$ of cholera toxin diluted in $1.5 \mathrm{ml}$ of PBS (0.15 M NaCl, $0.05 \mathrm{M} \mathrm{Na}_{2} \mathrm{HPO}_{4}, \mathrm{pH}$ 7.5). The abdominal wall was then closed and the animal was allowed to wake up. The duration of the challenge was five hours after which the rat was killed by cervical spine dislocation, the abdomen opened, and the loop dissected out. Net fluid secretion $(\mathrm{mg} / \mathrm{cm})$ was estimated by subtracting the weight of a control loop from that of the experimental animal loop. The AF preparation with $50 \%$ inhibition of fluid secretion was assigned an AF value of 1.0.

\section{STATISTICAL ANALYSES}

Data are presented as mean $(95 \%$ confidence intervals) unless otherwise stated. Variables were first examined for normal distribution using the Komolgorov-Smirnov test. Group means for non-normally distributed variables were compared using the Mann-Whitney test.
Group means of variables without significant deviation from normality were compared using the Student's $t$ test. Differences in proportions were assessed by $\chi^{2}$ tests (Pearson $\chi^{2}$ and Cochran's linear trend). Correlations were examined using Spearman's rho. A p value of 0.05 or less was considered significant. All calculations were made using SPSS version 7.5 software (SPSS Inc., Chicago, Illinois, USA).

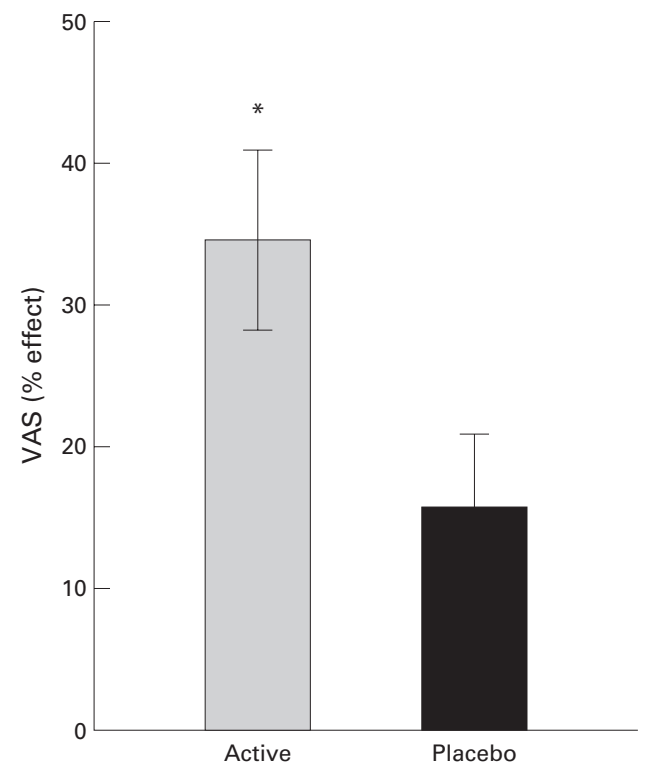

Figure 1 Subjective estimation of the effects of the two diets after four weeks of treatment, expressed as percentage improvement of the condition (VAS). Data are mean (SEM). * ${ }^{\star}$ Significant difference between the active and placebo groups $(p<0.05)$. VAS, visual analogue scale.

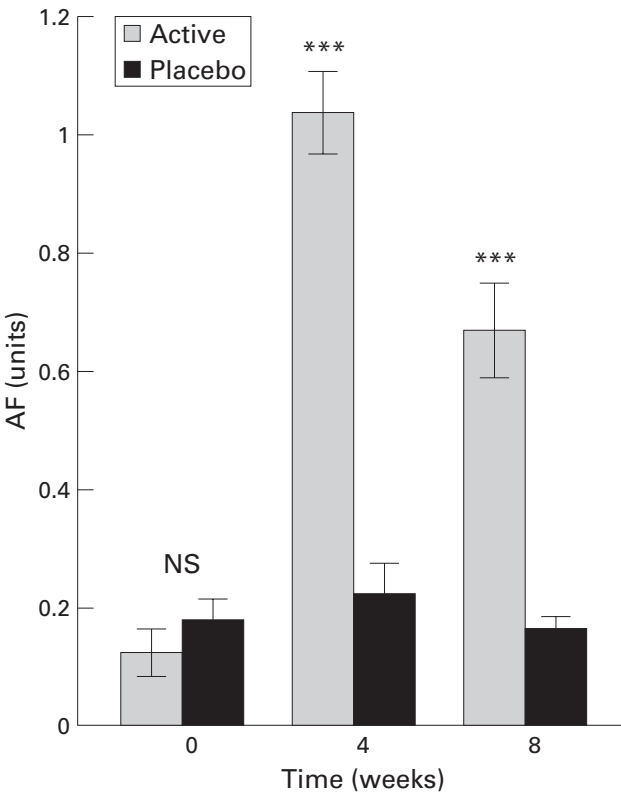

Figure 2 Plasma levels of antisecretory factor $(A F)$ in the two groups of patients at the start of the experiment, after four weeks of active or placebo diet, and after eight weeks (that is, four weeks after termination of the diets). Data are mean (SEM). ***Significant difference between groups $(p<0.001)$ at four and eight weeks. NS, not significant. 
Table 2 Histopathological rating of biopsies

\begin{tabular}{ll}
\hline I & $\begin{array}{c}\text { Few AF positive cells, no acute inflammatory } \\
\text { reaction }\end{array}$ \\
II & $\begin{array}{c}\text { Moderate number of AF positive cells, no acute } \\
\text { inflammatory reaction } \\
\text { Large number of AF positive cells, no acute } \\
\text { inflammatory reaction }\end{array}$ \\
III $\quad \begin{array}{c}\text { Moderate number of AF positive cells, acute } \\
\text { inflammatory reaction with ongoing crypt } \\
\text { destruction } \\
\text { Moderate number of AF positive cells, acute } \\
\text { inflammatory reaction with extensive crypt } \\
\text { destruction }\end{array}$ \\
V
\end{tabular}

\section{Results}

PATIENT RESULTS

Fifty of 53 patients completed the whole study: two patients in the placebo group withdrew from the study after 11 and 15 days, respectively, because of abdominal discomfort; one patient in the active group was excluded because of severe recurrence of his illness during the study. A detailed retrospective investigation of these three patients (anamnestic and laboratory values) provided no further information on why these patients terminated the study. A correlation between intake of placebo or active cereals and intestinal/clinical discomfort seems highly unlikely.

The distribution of the remaining patients was as follows, grouped according to the randomised double blind coding: of 26 patients given hydrothermally processed cereals, 16 were women (total ulcerative colitis $n=13$, distal ulcerative colitis $n=2$, Crohn's disease $n=1$; mean age 49.6 (SEM 5.1) years) and 10 were men (total ulcerative colitis $n=6$, distal ulcerative colitis $n=3$, Crohn's disease $n=1$; mean age 41.2 (5.8) years). Of the 24 patients in the pla-

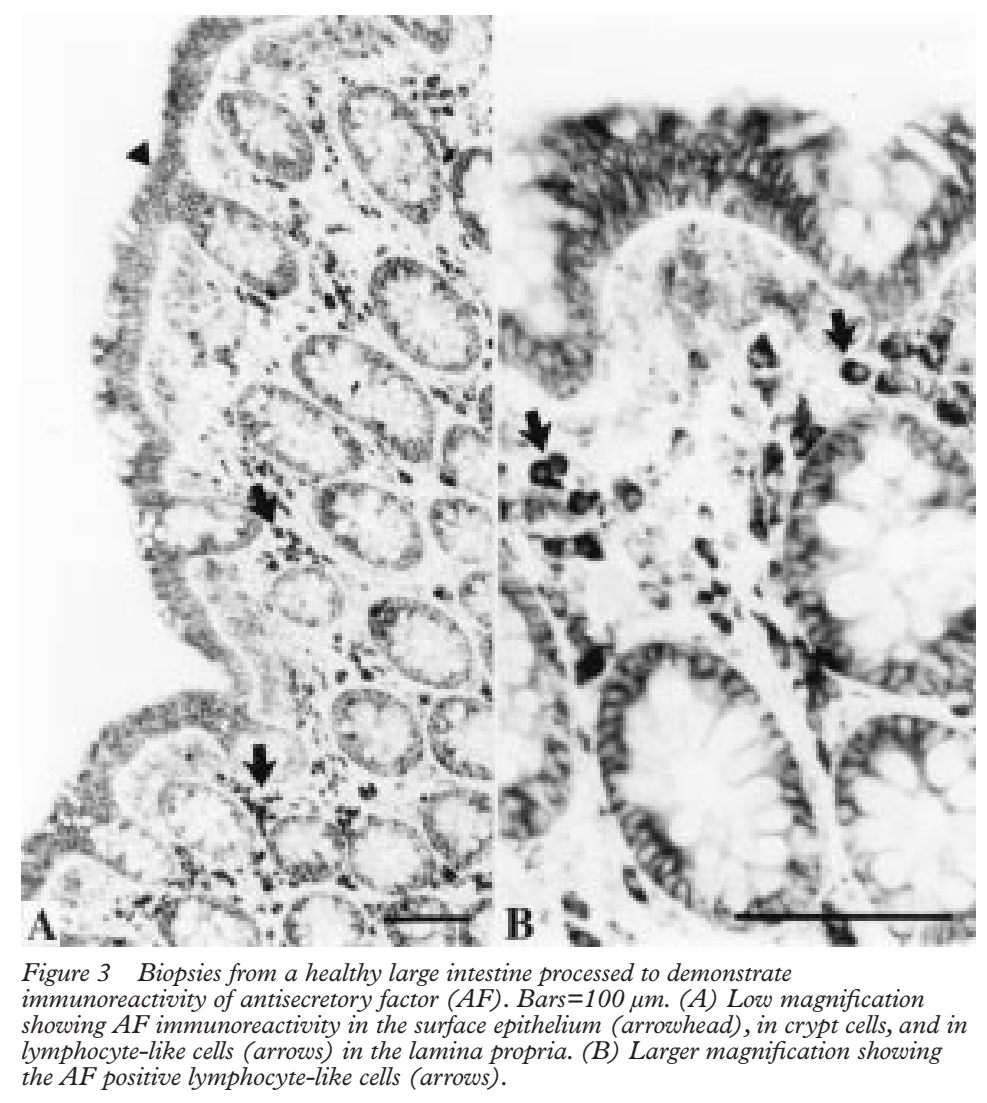

cebo group, 12 were women (total ulcerative colitis $\mathrm{n}=5$, distal ulcerative colitis $\mathrm{n}=7$; mean age 40.9 (3.7) years) and 12 were men (total ulcerative colitis $n=5$, distal ulcerative colitis $\mathrm{n}=6$, Crohn's disease $\mathrm{n}=1$; mean age 51.1 (4.5) years).

The clinical effects of the active and placebo diets were evaluated using the diaries kept by the patients during the two weeks before the study and for the four week study period. The hydrothermally processed cereals caused more solid stools and decreased the frequency of bowel movements compared with the placebo diet. The subjective estimates by the patients on the visual analogue scale (VAS), expressed as percentage change in subjective condition (fig 1), showed a significant difference between the active and placebo diets (active mean $+34.6 \%, \mathrm{n}=25$, one patient in the active group did not complete this evaluation; placebo mean $+15.4 \%, \mathrm{n}=24$, difference 19.2 , confidence interval 1.8-36.5; p<0.05). Chi-square tests were also performed on this material. Patients' estimations of the treatment effects were classified into three groups: (1) worse, (2) unchanged, or (3) improved, according to their VAS ratings. None in the active and one patient in the placebo group reported worsened symptoms, six in the active and 15 in the placebo group reported no change, while improvement was reported in 19 patients in the active group and in eight in the placebo group (Pearson $\chi^{2}$ 9.32, $\mathrm{df}=2, \mathrm{p}=0.009$; Cochran's linear trend $9.27, \mathrm{df}=1, \mathrm{p}=0.002$ ). Conclusively, irrespective of the statistical treatment of the patient material, the difference in VAS ratings between the active and placebo groups remained significant.

ANALYSIS OF AF LEVELS IN PLASMA

The results of the AF unit test (fig 2) demonstrated no difference between the active and placebo groups at the start of the experiment (day 0). At the end of the test period (four weeks), AF levels in the active group were greatly increased, resulting in a significant difference between the active and placebo groups $(p<0.001)$. AF values declined slowly after termination of active food intake. However, a significant $(\mathrm{p}<0.001)$ difference between the active and placebo groups still persisted four weeks after termination of the diet (that is, eight weeks after the start of the test).

At the start of the study, elevated levels of $\mathrm{C}$ reactive protein $(\mathrm{CRP})(>10 \mathrm{~g} / \mathrm{l})$ were observed in eight patients in the active group and in two in the placebo group, resulting in a significant difference $(p<0.05)$ between groups. There was no difference between groups in samples obtained four and eight weeks after the start of the study. There were no differences between groups in blood lipid values (total cholesterol, HDL cholesterol, and triglycerides), which were within normal limits in all patients (data not shown). 


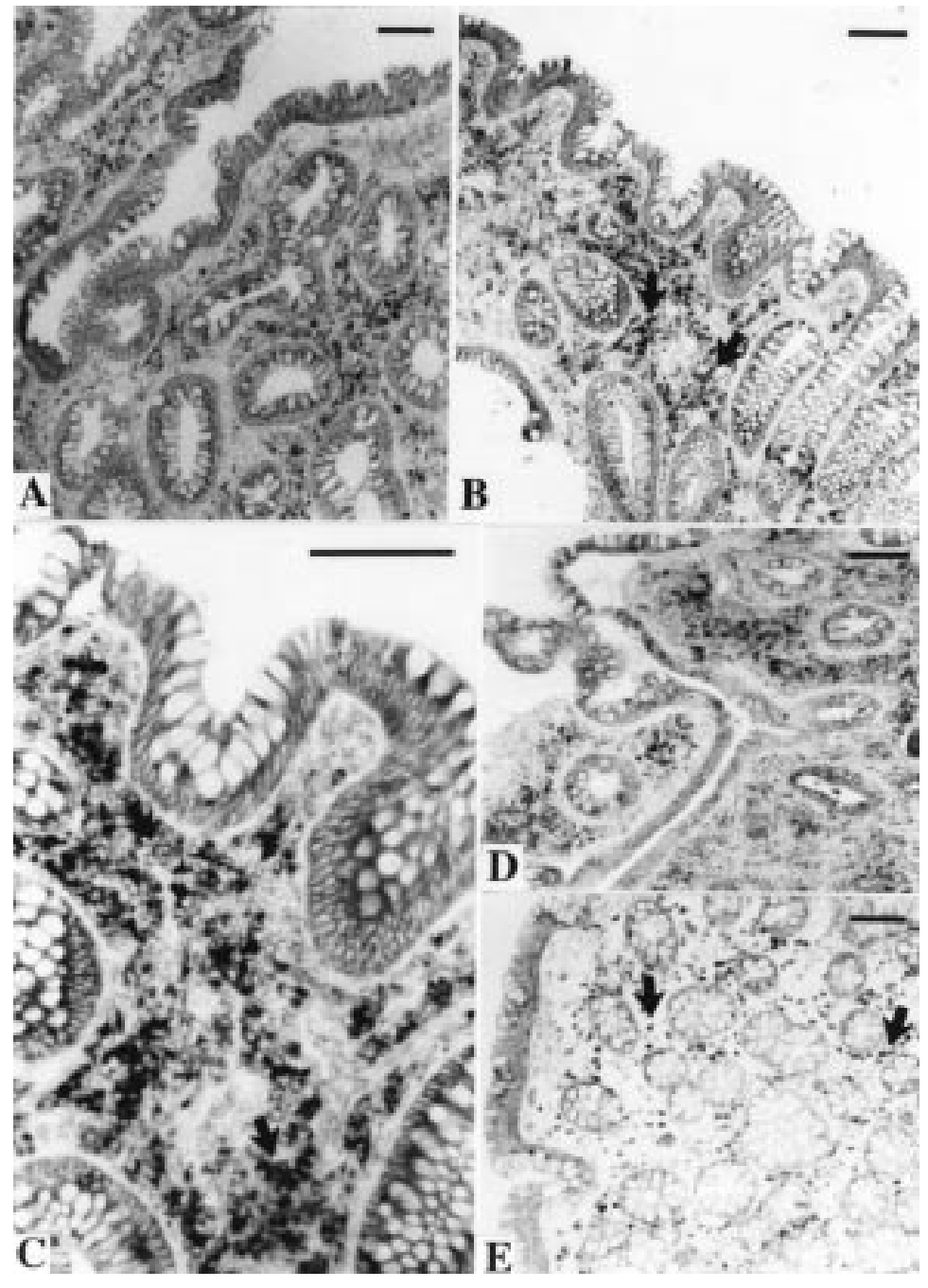

Figure 4 Rectal biopsies from patients with ulcerative colitis treated with the active diet. The sections were incubated with an antiserum against antisecretory factor $(A F)$. Bars $=100 \mu \mathrm{m}$. (A) Patient No 31 before the diet period. (B) Patient No 31 after the diet period. There appears to be more $A F$ positive cells (arrows) in the lamina propria after the diet period. (C) Larger magnification of (B), showing $A F$ positive cells (arrows) in the lamina propria. (D) Patient No 12 before the diet period. There are signs of an acute inflammatory reaction. Many small inflammatory cells show moderate $A F$

immunoreactivity. (E) The same patient after the diet period. In this biopsy there is no acute inflammation. Large AF positive cells (arrows) are evenly distributed within the entire lamina propria.
Table 3 Number of biopsies ( $n$ ) with various

histopathological ratings from patients with inflammatory bowel disease, before $(A)$ and after $(B)$ the diet period

\begin{tabular}{llllll}
\hline \multirow{2}{*}{$\begin{array}{l}\text { Rating of } \\
\text { biopsy }\end{array}$} & \multicolumn{2}{l}{ Active diet } & & \multicolumn{2}{l}{ Placebo diet } \\
\cline { 2 - 3 } \cline { 5 - 6 } & $A(n)$ & $B(n)$ & & $A(n)$ & $B(n)$ \\
\hline I & 9 & 7 & & 4 & 4 \\
II & 7 & 11 & & 8 & 8 \\
III & 4 & 6 & & 5 & 5 \\
IV & 2 & 0 & & 3 & 2 \\
V & 2 & 0 & & 3 & 4 \\
\hline
\end{tabular}

Table 4 Effect of treatment on extent of acute inflammation. Comparison of paired $A$ and $B$ rectal biopsies

\begin{tabular}{lcc}
\hline $\begin{array}{l}\text { Effect on acute } \\
\text { inflammation }\end{array}$ & Active diet $(n)$ & Placebo diet $(n)$ \\
\hline Worse & 0 & 5 \\
No change & 20 & 13 \\
Improved & 4 & 5 \\
\hline
\end{tabular}

staining was seen in relatively large mononuclear cells with a general appearance of lymphocytes. Staining of serial sections with various $\mathrm{T}$ cell antisera (CD3, CD4, CD8, CD20, CD45RO, and CD68; results not shown) indicated that the AF positive cells consisted of a subgroup of $\mathrm{T}$ cells and could be either CD4 or CD8 positive. However, a more definite classification of the AF positive cells remains to be done for both gross morphology and cell category.

In patients with acute inflammatory reactions, weak AF staining was also seen in many smaller inflammatory cells. Evaluation of the biopsies was based on the relative number of $\mathrm{AF}$ positive cells in the lamina propria and on the presence or absence of active inflammation with ongoing destruction of the crypts (fig 4).

Table 3 shows the morphological ratings of the patients before (A) and after (B) the diet period. To evaluate the effect of the treatment on acute inflammation in the individual case, the histology of A and B biopsies was compared and the extent of acute inflammation after the diet period classified as worse, unchanged, or improved (table 4). Analysis of the paired data in table 4 using the Pearson $\chi^{2}$ test revealed a significant difference between the active and placebo diets concerning the sections demonstrating acute inflammation after the diet period $\left(\chi^{2}\right.$ 6.578, $\left.\mathrm{df}=2, \mathrm{p}=0.037\right)$, while Cochran's linear trend did not reach statistical significance $(1.123, \mathrm{df}=1, \mathrm{p}=0.29)$. There were no significant differences in the relative number of AF positive cells between the $\mathrm{A}$ and B biopsies in any group. Furthermore, there was no correlation between AF levels in blood samples and the relative number of AF positive cells in the biopsies in any group. sections. Such material was excluded from the study. In 47 patients both biopsies were evaluated: of these 24 were from the active group and 23 from the placebo group.

In our ongoing research in healthy volunteers (unpublished), AF immunoreactivity in specimens from such subjects was found in the epithelial lining of the intestine and in mononuclear cells in the lamina propria (that is, similar to the results of cellular localisation demonstrated in fig 3). The most distinct

\section{Discussion}

Our study showed that AF can be induced in humans suffering from IBD using hydrothermally processed cereals, and that this increase in $\mathrm{AF}$ in blood is associated with a reduction in clinical symptoms. Thus a four week period of optimised, hydrothermally processed cereal diet led to subjective and objective improvements in the clinical course of IBD. In accordance with 
previous experimental studies in $\operatorname{rats}^{78}$ and pigs ${ }^{9}$ as well as field studies in pigs, ${ }^{34}$ it was found that a specific composition of the diet was followed by increased $\mathrm{AF}$ activity in plasma samples. Furthermore, an increase in AF activity correlated positively with patients' own subjective assessments of clinical improvement. Our limited study did not reveal any difference in the response to the diet between patients suffering from total or distal ulcerative colitis. A significant increase in the patient material is required to answer this clinically important question. The two patients with Crohn's disease who received the active diet both responded with significant improvement (VAS $+100 \%$ and $+50 \%$, respectively). Irrespective of the statistical methods used for analysis of the patient material, the difference between treatments remained significant when evaluated by symptom.

Randomisation gave a homogeneous distribution with regard to sex and age in the active and placebo groups. Except for one initial high CRP value in the active group, no difference in CRP values between the two groups was found during the course of the study. No effect on blood lipids was observed with either diet, indicating that the hydrothermally processed cereals did not affect lipid metabolism. However, these results should be evaluated with care as blood samples were not obtained under fasting conditions.

In accordance with previous studies in pigs ${ }^{10}$ the $\mathrm{AF}$ antiserum recognised surface and crypt epithelial cells and mononuclear cells in lamina propria in the biopsies from patients. These cells have the general appearance of large lymphocytes and are probably made up of a subgroup of T lymphocytes. Participation of T lymphocytes in Crohn's disease has been well documented $^{11}$ and so also the case for these cells in ulcerative colitis. ${ }^{12}$ However, we have been unable to find a single CD marker that conclusively identifies the entire AF positive population. The morphological similarities between AF positive cells and lymphoid cells is of major interest as various forms of immune phenomena are believed to play an important role in the pathogenesis of tissue damage both in Crohn's disease and in ulcerative colitis. ${ }^{13}{ }^{14}$ Furthermore, the use of immunohistochemistry in combination with in situ hybridisation in IBD may provide important information on the various factors involved in the genetics, ${ }^{15}$ aetiology, or pathogenesis of these diseases. ${ }^{16-18}$

In biopsies from patients receiving the active diet there was a tendency to a larger number of $\mathrm{AF}$ positive cells in the lamina propria and to a decrease in crypt destruction compared with biopsies from patients receiving the placebo diet. However, when considering only no acute inflammatory reaction versus acute inflammatory reaction, a difference was seen between the active and placebo diets, suggesting a positive effect of the diet on inflammatory status. These data, however, should be interpreted with caution as the total number of patients in the study was comparatively small and the incidence of acute inflammatory changes in the biopsies was low in the total material. This was also reflected in the statistical analyses as the Pearson $\chi^{2}$ test revealed a significant difference between groups while the linear trend test failed to reach statistical significance.

We did not find any correlation between $\mathrm{AF}$ activity in blood samples and staining of $\mathrm{AF}$ positive cells in the rectal biopsies analysed by immunohistochemistry. Previous northern blot and in situ hybridisation analyses indicated that AF mRNA is expressed in several peripheral tissues in addition to the initially described expression in the pituitary gland. ${ }^{10}$ We do not know the source of the dietary induced AF in blood but it is unlikely that serum AF reflects $\mathrm{AF}$ produced in the rectum. The increase in serum $\mathrm{AF}$ in response to the active diet might tentatively be due to AF synthesis in other parts of the intestinal tract, for example the small intestine. Further studies are needed to clarify this question.

In conclusion, this study demonstrates the possibility of inducing AF in IBD patients by supplementing their conventional diet with hydrothermally processed cereals. The increase in $\mathrm{AF}$ in plasma samples was associated with clinical improvement. Further studies are warranted to elucidate the role of $\mathrm{AF}$ in human intestinal disease.

Financial support was provided by the AS Factor AB, Nectin $\mathrm{AB}$, the Swedish State under the LUA agreement (grant No I 33 913), and the Swedish Medical Research Council (grant No 12 611),

1 Johansson E, Lönnroth I, Lange S, et al. Molecular cloning and expression of a pituitary gland protein modulating intestinal fluid secretion. F Biol Chem 1995;270:20615-20

2 Johansson E, Jennische E, Lange S, et al. Antisecretory factor suppresses intestinal inflammation and hypersecretion. Gut 1997;41:642-5

3 Göransson L, Lange S, Lönnroth I. Post weaning diarrhoea: focus on diet. Pigs News Information 1995;16:89-91N.

4 Göransson L, Martinsson K, Lange S, et al. Feed induced lectines in piglets. Feed-induced lectines and their effect on post-weaning diarrhoea, daily weight gain and mortality. $\mathcal{F}$ Vet Med B 1993;40:478-84.

5 Lennard-Jones JE. Classification of inflammatory bowel disease. Scand $\mathcal{F}$ Gastroenterol 1989;24:2-6S.

6 Lange S. A rat model for an in vivo assay of enterotoxic Lange S. A rat model for an in vivo assay of enter
diarrhoea. FEMS Microbiol Lett 1982;15:239-42.

7 Lönnroth I, Lange S. Intake of monosaccharides or amino acids induces pituitary gland synthesis of proteins regulating intestinal fluid transport. Biochim Biophys Acta 1987;925:117-23.

8 Lönnroth I, Lange S, Skadhauge E. The antisecretory actors: inducible proteins which modulate secretion in the small intestine. Comp Biochem Physiol 1988;90:611-17.

9 Lange S, Lönnroth I, Skadhauge E. Effects of the antisecretory factor in pigs. Pflügers Arch 1987;409:328-32.

10 Lange S, Jennische E, Johansson E et al. The antisecretory factor-synthesis and intracellular localisation in porcine tissues. Cell Tissue Res 1999;296:607-17.

11 Powrie F. T cells in inflammatory bowel disease: protective and pathogenic roles. Immunity 1995;3:171-4.

12 Fuss IJ, Neurath $M$, Boirivant $M$, et al. Disparate $C D+4$ lamina propria (LP) cytokine secretion profiles in inflammatory bowel disease. F Immunol 1996;157:1261-70.

13 Podolsky DK. Inflammatory bowel disease (first of two parts). N Engl f Med 1991;324:928-37.

14 MacDermott RP, Stenson WF. Alteration of the immune system in ulcerative colitis and Crohn's disease. Adv Immunol 1988;42:285-328.

15 Duerr RH. Genetics of inflammatory bowel disease. Inflamm Bowel Dis 1996;2:48-60.

16 Miyamoto $\mathrm{H}$, Tanaka $\mathrm{T}$, Kitamoto $\mathrm{N}$, et al. Detection of immunoreactive antigen, with a monoclonal antibody to measles virus, in tissue from a patient with Crohn's disease. $\mathcal{F}$ Gastroenterol 1995;30:28-33.

17 Wakefield AJ, Sim R, Akbar AN, et al. In situ immune responses in Crohn's disease: a comparison with acute and persistent measles virus infection. F Med Virol 1997;51:90100 .

18 Thompson NP, Montgomery SM, Pounder RE, et al. Is measles vaccination a risk factor for inflammatory bowel disease? Lancet 1995;345:1071-4. 